\title{
Economía y equidad en urgencias y emergencias
}

\section{Economics and equity in urgency and emergency care}

\author{
E. Moreno-Millán' ${ }^{1}$, M. Tejedor-Fernández², J.M. Torres-Murillo ${ }^{3}$, J.M. García-Torrecillas ${ }^{4}$, \\ M. Cid-Cumplido ${ }^{1}$, J. Villegas-del Ojo ${ }^{1}$, A.M. García-Fernández ${ }^{1}$
}

\section{RESUMEN}

La sostenibilidad financiera de los sistemas sanitarios públicos (SSP) está amenazada por el crecimiento poblacional, la mayor prevalencia de procesos crónicos y discapacidades, la inequidad residual en el acceso y utilización de los recursos, el coste nulo en la prestación y la crisis económica mundial.

Los servicios de Urgencias y Emergencias (SUE) son uno de los más demandados -sin relación con el modelo de salud- porque la enfermedad asienta en áreas sociodemográficas menos favorecidas, se mantienen inequidad, hiperconsumo y capacidad de decisión más ligada al usuario. El producto sanitario de los SUE es múltiple y con líneas de producción diversas que dificultan su medición. En esta revisión se analiza la necesidad de implantar herramientas de medida en los SUE, donde existen altos costes directos -fundamentalmente estructurales-y otros variables relacionados con la actividad, donde el coste marginal es superior al medio y sin economía de escala en estas intervenciones. Se estudian, asimismo, los posibles mecanismos de coparticipación privada en la financiación de la oferta de los SUE, se muestran sus ventajas e inconvenientes y se concluye que no son recomendables -por su escasa capacidad recaudadora y disuasoria- por lo que son necesarios cambios estratégicos fundamentales en la gestión de estos recursos.

Palabras clave. Urgencias. Emergencias. Economía de la salud. Costes. Gestión. Equidad.

\begin{abstract}
The financial sustainability of public health systems (PHS) is currently threatened by population growth, increased prevalence of chronic conditions and disabilities, inequality in access and use of resources, zero cost delivery and global economic crisis.

The emergency department (ED) is one for which demand is highest - without relation to the health model - because disease becomes established in disadvantaged socio-demographic areas and inequalities, hyperconsumption and decision making more closely linked to the user are maintained. The medical device of ED is a multiple one and its diverse product lines make it difficult to measure.

This review discusses the need to deploy measurement tools in $\mathrm{ED}$, where there are high direct costs - primarily structural - and other variables related to the activity, where the marginal cost is higher than the average and there is no economy of scale in such interventions. The possible mechanisms of private copayment in financing the supply of EDs are also studied, showing their advantages and disadvantages, with the conclusion that they are not recommendable - due to their scarce fund raising and deterrent capacity, which is why fundamental strategic changes in the management of these resources are needed.
\end{abstract}

Key words. Urgencies. Emergencies. Health economics. Costs. Management. Equity
1. Servicio de Medicina Intensiva. Hospital "Santa Bárbara». Puertollano. España.

2. Servicio de Documentación y Calidad. Hospital "Reina Sofía». Córdoba. España.

3. Servicio de Cuidados Críticos y Urgencias. Hospital "Reina Sofía». Córdoba. España.

4. Servicio de Cuidados Críticos y Urgencias. Complejo Hospitalario "Torrecárdenas».

Almería. España.

\author{
Correspondencia \\ E. Moreno Millán \\ Servicio de Medicina Intensiva \\ Hospital «Santa Bárbara» \\ Avda. Malagón, s/n \\ 13500 Puertollano. España. \\ E-mail: emormill@hotmail.com
}




\section{INTRODUCCIÓN}

Todos los estados de la Unión Europea (UE-27) donde la sanidad -aunque con diferentes modelos- es el servicio público más valorado, tienen dificultades para contener el aumento progresivo del gasto público en salud (GSPb) y conseguir su sostenibilidad financiera, como consecuencia del crecimiento demográfico, la mayor prevalencia de procesos crónicos y discapacidades y la crisis económica mundial ${ }^{1}$.

El aseguramiento es una respuesta racional ante el riesgo y la incertidumbre de los ciudadanos para enfermar, sin conocer el momento ni la intensidad de los procesos. Derivados de la existencia de un mecanismo de seguro ante fenómenos adversos, pagado fiscalmente por todos los individuos, surgen comportamientos oportunistas o abusivos -como el riesgo moral (moral hazard)- que se traducen en hiperconsumos y que provocan desequilibrio entre oferta de recursos y demanda de salud $^{2,3}$.

Los tributos que soporta el sector público no mantienen relación entre la contribución y el servicio consumido y la ausencia de coste de oportunidad -debida al precio nulo en el momento de la prestación- provoca una sobredemanda no originada por una verdadera necesidad de salud.

\section{ECONOMÍA Y URGENCIAS}

En los últimos 30 años existe una creciente demanda asistencial en los servicios de urgencias y emergencias (SUE) de todo el mundo, que no obedece, habitualmente, a necesidades objetivas de salud sino a procesos socialmente arraigados por razones diversas ${ }^{4}$. Progresivamente se requiere mayor calidad en la atención que, unida al avance en medios diagnósticos y terapéuticos y su consiguiente encarecimiento, explica el incremento del coste. La actual situación de crisis económica mundial ha colocado también a los servicios de salud en el centro del debate.

Los SUE se caracterizan por accesibilidad y disponibilidad total y mantienen ac- tivos los recursos necesarios incluso cuando no estén prestando ningún servicio. Es lo que se conoce como un "bien público»: todos los individuos lo consumen aunque nunca lleguen a utilizarlo. En este sentido, es necesario proveer unos gastos estructurales -su mayor parte suelen ser costes fijos- que constituyen el componente más importante, aunque existe otro paquete, relacionado con la casuística y la actividad asistencial, donde se incluyen los in-put que colaboran en el proceso de producción y que, por tanto, son costes variables ${ }^{5}$.

Para hacer sostenible esta prestación, caracterizada por demanda creciente en número y calidad, la estrategia clave es la búsqueda de la eficiencia, que relaciona la eficacia con los costes de producción. Desde un abordaje macro, los SUE disponen de información suficiente para conocer "cuánto cuestan" globalmente pero, para poder gestionar eficientemente estos servicios, es necesario desglosar la información de los procesos asistenciales y de la génesis de su coste.

También tiene importancia conocer el coste de los procesos clínicos que se atienden en los SUE porque aportan una ayuda extraordinaria para perfeccionar el conocimiento sobre la casuística y las actividades realizadas en el estudio y tratamiento de cada proceso. Su análisis permite decidir prioridades en formación, orientar y mejorar la elaboración de los protocolos, evaluar la calidad asistencial, analizar la eficiencia y utilizar un lenguaje común con directivos y gestores sanitarios a los que facilita la toma de decisiones a través del análisis de coste-oportunidad, coste-efectividad y coste-beneficio, orienta en estrategias productivas y resulta imprescindible para hacer una óptima facturación.

La medición del producto sanitario ha sido -y sigue siendo- un reto metodológico por su dificultad y, para ello, se han empleado muchos sistemas de clasificación de pacientes ${ }^{6}$ (Tabla 1 ).

En los SUE no se ha implantado definitivamente ninguna agrupación de pacientes relacionada para medir el producto. Atienden una gran variedad de procesos (multi- 
producto: desde patologías banales hasta críticas) y se le obliga a utilizar -y, por lo tanto, a consumir- una extensa diversidad de recursos. Esta actividad asistencial es una de las más importantes en todos los países del mundo, por el volumen económico que representa y porque, generalmente, constituye la vía de acceso al sistema de salud.

Tabla 1. Sistemas de medición del producto sanitario.

\begin{tabular}{l}
\hline Medida del producto hospitalario \\
\hline Grupos relacionado con el diagnóstico (GRD) \\
Disease staging (DS) \\
Patient management categories (PMC) \\
\hline Medida del producto ambulatorio \\
\hline Ambulatory visit groups (AVG) \\
Ambulatory patient groups (APG) \\
Adjusted clinical groups (ACG) \\
\hline Medida del producto en urgencias y emergencias \\
\hline Emergency diagnostic groups (EDG) \\
Urgency related groups (URG)
\end{tabular}

A pesar del enorme impacto en el consumo, el análisis de costes no goza de gran presencia en la bibliografía. Ética y profesionalmente la investigación debe dirigirse hacia el conocimiento del coste y cómo se genera éste en cada tipo de proceso clínico. La Sociedad Española de Medicina de Urgencias y Emergencias (SEMES) constituyó, en 1998, un grupo de trabajo de costes que hizo una interesante aproximación al tema, en un intento de proponer una clasificación de pacientes por isoconsumo ${ }^{7,8}$, y se planteaba dos grandes objetivos generales: desarrollar un modelo de cálculo de costes de los procesos clínicos de los SUE y agrupar éstos en categorías de isoconsumo que permitieran la comparación entre servicios (benchmarking) en términos de eficiencia y su utilización como instrumento de financiación.

Las fases del proyecto se muestran en la tabla 2: se superaron las fases 1 y 2 y se inició la 3, comenzando el estudio en el Hospital Universitario "Reina Sofía». Se realizó un análisis retrospectivo de las patologías más frecuentes atendidas en el SUE, excluyéndose los procesos de las áreas de Pediatría y Ginecología. Para ello, se extrajo una muestra aleatoria del total de 102.097 pacientes, con error del $5 \%, \mathrm{p}<0,05$ y estimación inicial del $50 \%$, por lo que se utilizó el programa C-4 Study Design Pack y se obtuvieron 384 casos. Para identificar al proceso clínico se codificó el diagnóstico principal -utilizando la Clasificación Internacional de Enfermedades ( $9^{\mathrm{a}}$ revisión, Modificación Clínica (CIE-9 MC) ${ }^{9}$ - entendiendo como tal la causa que motivara la demanda asistencial urgente. Los cinco procesos clínicos más frecuentes fueron: esguince de tobillo, cólico nefrítico, insuficiencia cardíaca congestiva, vértigo periférico y crisis de ansiedad. Se diseñó un mapa de actividades (Fig. 1) que identificaba e integraba las relaciones entre actividades principales y secundarias por medio de un flujograma. Se calcularon e imputaron los costes directos a las actividades realizadas en cada proceso clínico (Tabla 3). La diferencia en el coste total de los procesos analizados estribaba en los directos, ya que el resto se imputó lineal y homogéneamente por cada asistencia y fue el mismo para todos los procesos. La patología con mayor coste directo fue la insuficiencia cardiaca congesti- 
va, que llegó a duplicar a las que le seguían (cólico nefrítico y esguince de tobillo), cuadruplicar al vértigo periférico y sextuplicar a la crisis de ansiedad, que fue la de menor coste. Es decir, la herramienta se mostró útil para discriminar las diferencias

Tabla 2. Fases del proyecto de cálculo de costes de los procesos clínicos en los SUE.

- Diseño del método de cálculo de coste por proceso clínico para hacer posible su uso en cualquier nivel de la asistencia urgente (Hospitalarios, atención primaria y prehospitalarios)

- Validación del método con una serie de procesos clínicos

- Aplicación del modelo, para el cálculo del coste estándar, de aquellos tipos de procesos que supusieran el $80 \%$ de las asistencias

- Determinación de una unidad relativa de valor para el coste de los procesos clínicos, que se denominaría VAU (Valor de la asistencia urgente)

- Clasificación de los procesos en categorías isoconsumos y su posterior codificación en Grupos Isoconsumo en Urgencias (GIU)

Tabla 3. Cálculo e imputación de costes directos a actividades en cada proceso clínico.

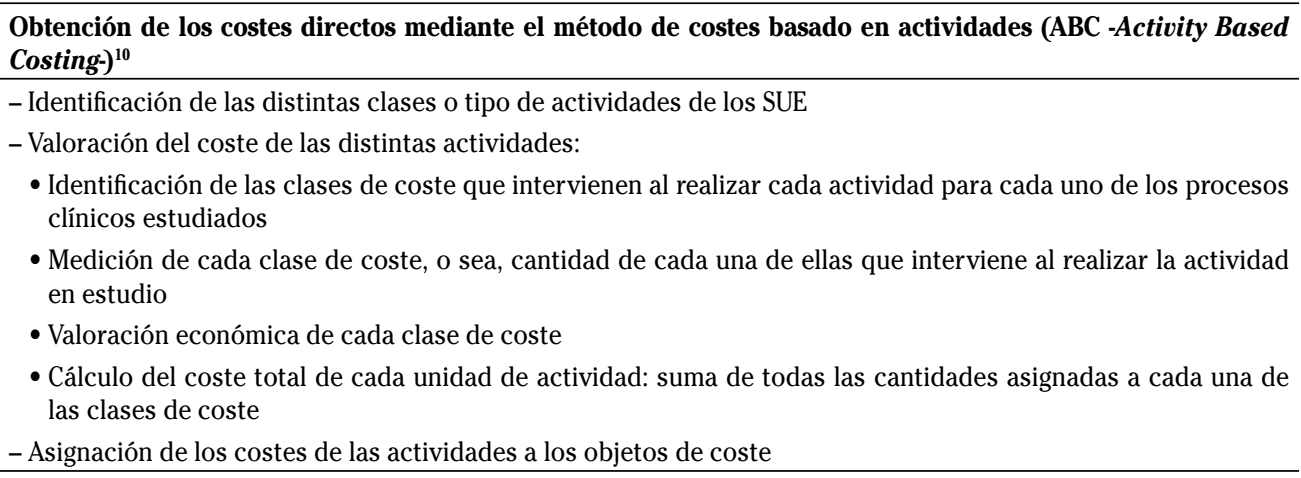
Imputación de los costes semidirectos, indirectos y repercutidos mediante el sistema de contabilidad analítica del hospital

Puede afirmarse que el abordaje del cálculo de costes en los SUE presenta importantes ventajas respecto a los métodos convencionales:

- Mejora la imputación y un conocimiento más preciso de ellos, de manera que facilita su evaluación y seguimiento y proporciona información real y adaptación al proceso de decisión clínica basado en protocolos.

- Al hacer confluir protocolos clínicos con actividades, propicia el análisis, la discusión y la evaluación interdisciplinar sobre el valor añadido y la efectividad de cada objetivo de coste, en términos de eficiencia clínica y económica, y constituye un instrumento potente en los programas de mejora de la calidad ya que resulta eficaz para evaluar la eficiencia de los distintos protocolos clínicos y establecer cuál de ellos incluye la secuencia óptima de operaciones o, incluso, para crear uno nuevo mejor basado en las aportaciones de cada una de las actividades al resultado final.

- El mayor grado de corrección y estandarización metodológicas permite la reproductibilidad del modelo y posibilita el benchmarking entre centros sanitarios. 


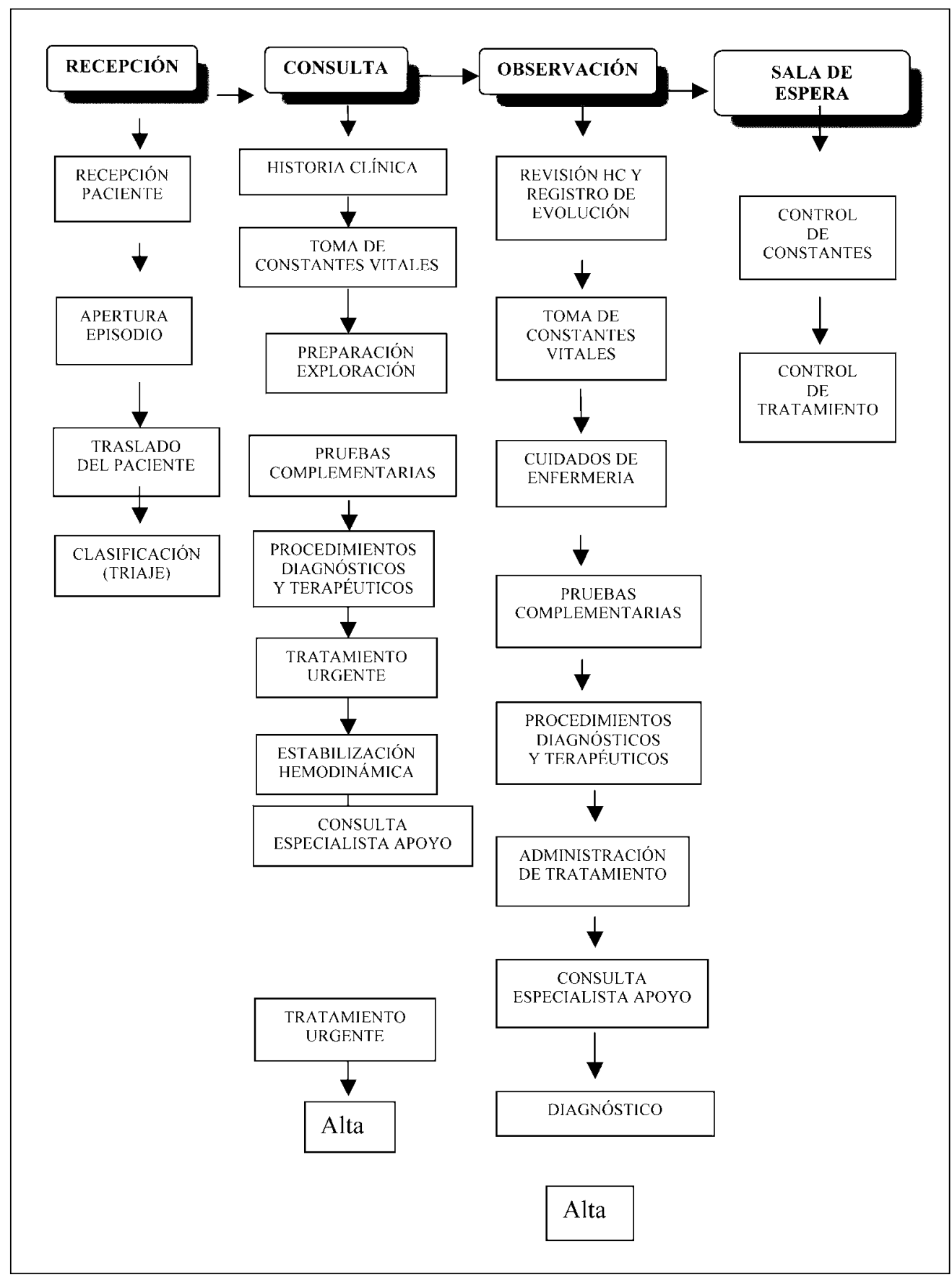

Figura 1. Mapa de actividades de los servicios de urgencias hospitalarios. 
En España no ha podido establecerse aún un sistema de clasificación de pacientes por isoconsumo en los SUE, por lo que no puedan hacerse comparaciones precisas de eficiencia entre servicios, y las posibilidades de introducir elementos para hacer una gestión clínica óptima -en base a los costes que generan las distintas actividades- no están disponibles. Mientras tanto, se investiga el modo de llegar al coste por paciente, o sea, el consumo de servicios que genera una persona en sus respectivos contactos con su servicio de salud. Esto debe animar a los responsables de los SUE a avanzar en este campo, pues las diferencias entre los usuarios de estos servicios es extraordinaria: basta reflexionar un poco sobre una posible facturación a multifrecuentadores.

El avance en la informatización de los procesos asistenciales, en todos los ámbitos de la sanidad, supone una herramienta básica para solucionar estos problemas. Es preciso diseñar correctamente la información que se vaya a utilizar, pero ahora se dispone de técnica y herramientas para introducir lo expuesto en soporte informático, de forma que se puede obtener el coste directo de cualquier episodio urgente. Además, es factible desglosar ese coste por áreas, actividades, etc, y hacer benchmarking con otros servicios.

Parece llegado el momento de tomar iniciativas y, con la ayuda de los departamentos económicos de las organizaciones sanitarias, abordar la tarea de conocer científicamente cómo se genera el coste de la asistencia urgente y, por tanto, el gasto. Esto redundará finalmente en beneficio de pacientes, autoridades y profesionales al incrementar competencias en un momento donde existe la posibilidad de que la atención inicial se realice siempre por un especialista en Medicina de Urgencias.

\section{EQUIDAD Y URGENCIAS}

Conseguir la equidad en el acceso y la utilización de los recursos de salud es una preocupación básica en los países que disponen de un sistema sanitario público
(SSP). Es fundamental el papel que estos servicios desempeñan reduciendo los problemas desencadenados por las desigualdades. La equidad horizontal ("igual acceso para igual necesidad» e «igual utilización para igual necesidad") independiza la oferta de la capacidad de pago, sexo, raza, morbilidad, residencia, etc, aunque en muchas ocasiones no es el paciente quien decide su consumo sanitario, sino que se delega la elección en el profesional para determinar la cantidad de recursos asignados a cada individuo. Para cuantificar la inequidad se calcula la diferencia entre el consumo sanitario real y la necesidad de atención médica, una vez clasificados los individuos en función de su renta mediante curvas de concentración (índice de Wagstaff y Van Doorslaer $)^{11}$.

En el SSP español se ha estudiado la posible existencia de inequidad horizontal en la prestación de determinados servicios (consultas a médico general y especialista, ingreso hospitalario y atención urgente) y se ha comprobado que la frecuencia de visitas al especialista tiene un patrón distinto a las consultas a generalistas y a los ingresos, en relación con la renta ${ }^{12}$. Existe una cierta concentración de la necesidad en los tramos inferiores de la escala social, no hay diferencias en los tiempos de acceso a consultas y no se observa una relación directa entre el tiempo medio y el porcentaje de visitas efectivas ante situaciones de necesidad similar, pero sí se aprecian "proricos" en las listas de espera (LE) hospitalarias, con mayores estancias en los estratos más bajos ${ }^{13}$. Se comprueba también que la desigualdad favorece a individuos de menos recursos en el generalista y que ocurre lo contrario en el especialista ${ }^{14,15}$. Con respecto a la atención urgente, se demuestra una inequidad "pro-ricos" en su acceso y utilización, con resultados comparables a otros países europeos ${ }^{16-18}$.

La existencia de inequidad en un modelo socialmente deseable, con incremento progresivo de su demanda, costes directamente relacionados con la actividad $-\mathrm{y}$, por tanto, con el crecimiento poblacionalen un escenario de crisis económica y de 
dudosa sostenibilidad financiera del SSP, obligan a debatir la posible introducción de mecanismos de cofinanciación privada que, asimismo, ejerzan un poder disuasorio en el empleo de estos recursos. En el caso sanitario, el copago es la participación del paciente en el coste del servicio durante su prestación. Existen muchas modalidades que pueden aplicarse, global o individualmente, en dependencia del enfermo o del producto $^{19}$.

Se han vertido algunas opiniones respecto al copago -muchas de ellas sin sustento científico- que suelen confundir a los ciudadanos, pero también a los profesionales: ¿hay que introducir copagos en nuestra atención sanitaria? ¿qué ventajas y qué inconvenientes presentan? ¿cómo y cuánto se modifica el empleo de un servicio y el de los posibles que se utilicen como alternativos? ¿son realmente eficientes en lo económico y efectivos en lo clínico? ¿inducen inequidad en el acceso o en la fiscalidad? ¿se pueden producir diferencias entre distintos grupos socioeconómicos? ¿se puede provocar empeoramiento de la salud, individual o colectiva? ¿reducen o disuaden del consumo por igual? ¿hay otras fórmulas alternativas? ¿qué costes de transacción conllevan?

Aun cuando parecen evidentes algunos de los beneficios que los copagos pueden originar, también se han expresado muchos efectos adversos. En este sentido, se ha apuntado que las circunstancias más adecuadas para su utilidad serían que la elasticidad-precio de la demanda no fuese nula, que afectara especialmente a productos de bajo coste-efectividad, que el acceso a los ciudadanos de menor renta o mayor necesidad estuviese garantizado y que los costes de transacción permitieran su implantación.

Pero, ¿cómo y en qué forma altera un copago el consumo de un producto? ¿cuál es su efecto asumiendo la existencia de incentivos? ¿cómo responden los individuos, especialmente los menos agraciados y enfermos, en función de su renta? ¿podría repercutir la dificultad de acceso sobre la salud del individuo o de la población y mantenerse a medio-largo plazos? ¿hay alguna alternativa que produzca el mismo bienestar con menor coste...?

La teoría económica afirma que el copago debería ser más bajo para los servicios con menor elasticidad-precio, mayor efectividad o sin alternativas. Si lo que se pretende es disminuir la sobredemanda, debería aplicarse sobre las más elásticas, pero si se busca incrementar la recaudación habría que hacerlo sobre las de menor elasticidad. Existe escasa evidencia sobre el resultado de los copagos en la salud, pero se ha comprobado su empeoramiento en la población más vulnerable y en las rentas más bajas, ya que la productividad marginal de un servicio es mayor cuanto peor es el nivel de salud y también la reducción del consumo, pero no la adecuación de la respuesta clínica ni el coste total asistencial, y parece afectarse el acceso de todos los pacientes, sean o no hiperconsumidores, incluso en recursos preventivos, lo que provocaría externalizaciones negativas en la salud comunitaria ${ }^{20}$.

Unas de las áreas de gestión de los SSP cuya alta frecuentación, escasa competitividad y elevado coste le confieren unas determinadas peculiaridades son los SUE. Estos recursos se utilizan excesivamente como entrada al sistema, constituyendo excepciones a las normales relaciones de agencia imperfecta que presentan el resto de ofertas sanitarias: en los SUE las decisiones de acceso están en manos de los ciudadanos-pacientes en lugar de los profesionales, se retrasa la atención -lo que podría ser especialmente grave en los pacientes complejos- y se ha comprobado que algunos pacientes prefieren no acudir al hospital o incluso buscar posibilidades de sustitución aunque desconocen el precio real del copago ${ }^{21}$.

La eficacia no se ve incrementada con la participación financiera privada, presiona al paciente sin facilitarle adecuada información, no parece disminuir la duración ni el número de servicios cuando se ha conseguido acceder al sistema. En definitiva, se transfiere riesgo a los pacientes porque se sanciona más al enfermo que al sano y al marginado 
frente al de mayor renta y, si es ajeno a ella, modifica regresivamente su redistribución: es decir, los copagos son inequitativos ${ }^{19-21}$. Desde el punto de vista recaudatorio tampoco hay apenas evidencia empírica de un beneficio. Al contrario, los países de la UE27 y de la OCDE con cifras más elevadas de coparticipación en los costes tienen porcentajes más elevados de GSPb sobre sus PIB ${ }^{1}$. Además, los mecanismos necesarios para llevar a cabo la recaudación -que es mínima, a no ser que se produzca un reaseguramiento privado frente al copago- son complejos y conllevan altos gastos de transacción que, en muchas ocasiones, disuaden a los políticos de su introducción, fundamentalmente porque provocan insatisfacción profesional y ciudadana ${ }^{22-24}$.

Las posibles soluciones pasan por mejorar la eficiencia (hacer más con lo mismo), aumentar la financiación pública (hacer más con más), incrementarla de forma privada (copagos) o racionar las prestaciones (listas de espera). Es evidente que todavía estamos lejos de conseguir, al menos en España, una situación menos ineficiente de nuestro SSP (uso inapropiado de ingresos, estancias y tecnología; excesivo gasto farmacéutico, escasa coordinación de la gestión hospitalaria con la primaria, inexistencia de red socio-sanitaria, no traslado de riesgos a los agentes, etc) ${ }^{25,26}$. El racionamiento de las prestaciones sólo puede darse en sistemas donde la demanda, aunque alta, obedece a una necesidad real de salud y donde existe una oferta adecuada de proveedores, lo que no es el caso de España. Las LE continúan siendo el peor indicador social de nuestro modelo de salud, especialmente cuando su tratamiento se realiza mediante peligrosas actuaciones a destajo, alejadas de la calidad y la satisfacción imprescindibles en un servicio público.

En conclusión, no existe una solución clara para el problema de la escasez presupuestaria: hay que sensibilizar, concienciar y responsabilizar a políticos, gestores, profesionales y ciudadanos para que tengan conocimiento verdadero de la diferencia entre demanda y necesidad. Y cuando la primera supere a la oferta, sepamos buscar la mejor alternativa, que no parece ser ni el inefectivo e inequitativo copago ni las inhumanas restricciones al acceso ${ }^{27}$. Introducir la coparticipación en los actuales SUE de nuestro SSP se acompañaría de un importante grado de insatisfacción social, escasa posibilidad recaudatoria con elevados gastos de transacción y disuasión parcial de enfermos que buscarían otros accesos sustitutorios -donde el coste marginal sería más elevado- y conduciría a probable empeoramiento del estado de salud. Ni clínica ni económicamente-ni mucho menos, socialmente- parece rentable esta herramienta financiera: serían precisos amplios estudios, de difícil y peligrosa realización, para poder afirmar tajantemente estas hipótesis. En el escenario español de crecimiento demográfico, crisis económica, inequidad y difícil sostenibilidad financiera del SSP se hace necesario aceptar alternativas inteligentes y cambios estructurales en un nuevo modelo estratégico de gestión.

\section{BIBLIOGRAFÍA}

1. OECD Health Data (PuBlic health expenditure), OECD, 2009.

2. Pauly M. The economics of moral hazard: comment. Am Econ Rev 1968: 58: 531-537.

3. ZWEIFEL P, MANNING WG. Moral hazard and consumer incentives in health care. En: Handbook of Health Economics, North-Holland, 2000.

4. Moreno-Millán E. Atención sanitaria urgente y demanda social: reflexiones sobre la elección del modelo de respuesta. Emergencias 1995; 7 : 108-115.

5. Bamezai A, Melnick G. Marginal cost of emergency department outpatient visits: an update using California data. Med Care 2006; 44 : 835-841.

6. Prados A, Marión J, Tejedor M, Moreno E. Herramientas de medida del producto sanitario en la asistencia a urgencias y emergencias. Todo Hospital 2002; 187: 322-327.

7. TeJedor M, Jiménez L, Bandera JJ. El coste de los procesos clínicos en los Servicios de Urgencias y Emergencias. Emergencias 1998, 19: 393-405.

8. Torres JM, Jiménez L, Tejedor M, Keller I, Temes JL. Coste por proceso en Urgencias. Todo Hospital 1999; 157: 383-390. 
9. International classification of diseases, 9th CM, World Health Organization, 1994.

10. Tejedor M, Jiménez Murillo L, Torres Murillo JM, Bandera GonzÁlez JJ, Arroyo MuÑoz JL, BuFoln Galiana A et al. El sistema $\mathrm{ABC}$ en el análisis del coste de los procesos clínicos en las unidades de urgencias y emergencias. Rev Cal Asist 1999; 14: 312-320.

11. Van Doorslaer E, Masseria C, Koolman X for the OECD Health Equity Research Group. Inequalities in access to medical care by income in developed countries. Can Med Association J 2006; 174: 177-183.

12. Regidor E, Martínez D, Astasio P, Ortega P, Calle ME, Domínguez V. Asociación de los ingresos económicos con la utilización y la accesibilidad de los servicios sanitarios en España al inicio del siglo XXI. Gac Sanit 2006; 20: 352359.

13. Murillo C, Carles N. Diseño de indicadores de capacidad adquisitiva de los usuarios para mejorar la racionalidad y la equidad en la financiación sanitaria. Ministerio de Sanidad y Consumo y CRES, Madrid-Barcelona, 1999.

14. URBANOS RM. La prestación de los servicios sanitarios públicos en España: cálculo y análisis de la equidad horizontal interpersonal para el período 1987-1995. Hacienda Pública Española 2000; 153: 139-160.

15. Van Doorslaer E, Koolman X. Explaining the differences in income-related health inequalities across European countries. Health Economics 2004; 13: 609-628.

16. URbanos RM. Measurement of inequity in the delivery of public health care: evidence from Spain (1997). Documento de trabajo 2001/15, FEDEA, Madrid, 2001.

17. URbANos RM. Equidad en el acceso a los servicios sanitarios: una revisión de la evidencia disponible. Inguruak 2007; 44: 183-192.
18. GaRcía P, LóPEz A. The evolution of inequity in the access to health care in Spain: 1987-2001. Documento de trabajo 756, Departamento de Economía y Empresa, Universidad Pompeu Fabra, Barcelona, 2006.

19. Puig-Junoy J. Los mecanismos de copago en servicios sanitarios: cuándo, cómo y por qué. Hacienda Pública Española 2001; 158: 105-134.

20. López-Casasnovas G, Puig-Junoy J, Ganuza JJ, PlaNAS I. Los nuevos instrumentos de la gestión pública. Colección Estudios Económicos, núm. 31, Caja de Ahorros y Pensiones La Caixa, Barcelona, 2004.

21. Hsu J, Reed M, Brand R, Fireman BH, Newhouse JP, SELBy JV. Cost-sharing: patient knowledge and effects on seeking emergency department care. Med Care 2004; 42: 290-296.

22. Selby JV. Cost sharing in the emergency department - is it safe?, it is needed? N Engl J Med 1997; 336: 1750-1751.

23. Selby JV, Fireman BH, Swain BE. Effect of a copayment on use of the emergency department in a health maintenance organization. N Engl J Med 1996; 334: 635-641.

24. Reed M, Fung V, Brand R, Fireman B, Newhouse JP, SelBy JV et al. Care-seeking behaviour in response to emergency department copayments. Med Care 2005; 43: 810-816.

25. McConnell KJ. What do health savings account mean for the emergency department? Ann Emerg Med 2005; 46: 536-540.

26. Navarro V. Análisis para la Asociación de Federaciones para la Defensa de la Sanidad Pública. Salud 2000, núm. 33, 1991.

27. Moreno-Millán E. ¿Y si adaptáramos los servicios hospitalarios de urgencias a la demanda social y no a las necesidades de salud? Emergencias 2008; 20: 276-284. 
